Flyunt Viktor R., Flyunt Igor-Severyn S., Ruzhylo Sofiya V., Fihura Oksana A., Popovych Dariya V., Żukow Xawery. Relationships of urolithogenicity index with some components of urine composition in healthy old female rats. Test of ventromedial prefrontal cortex in context of making moral choices. Journal of Education, Health and Sport. 2021;11(10):357-372. eISSN 2391-8306. DOI http://dx.doi.org/10.12775/JEHS.2021.11.10.034

https://apcz.umk.pl/JEHS/article/view/JEHS.2021.11.10.034

https://zenodo.org/record/5847394

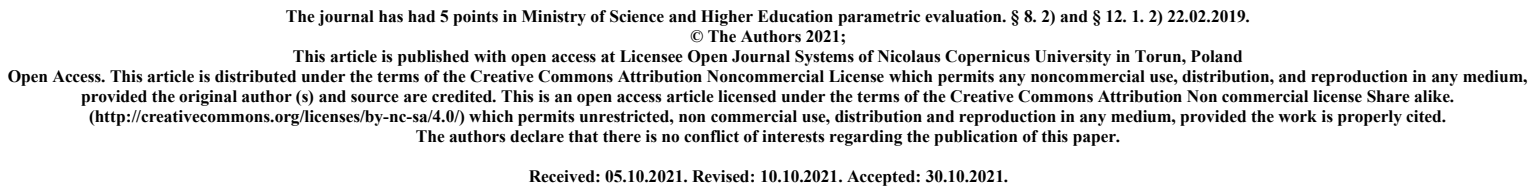

Received: 05.10.2021. Revised: 10.10 .2021 . Accepted: 30.10 .2021$.

\title{
RELATIONSHIPS OF UROLITHOGENICITY INDEX WITH SOME COMPONENTS OF URINE COMPOSITION IN HEALTHY OLD FEMALE RATS
}

\author{
Viktor R. Flyunt ${ }^{1,2}$, Igor-Severyn S. Flyunt ${ }^{3}$, Sofiya V. Ruzhylo ${ }^{3}$, Oksana A. Fihura ${ }^{3}$, \\ Dariya V. Popovych ${ }^{4}$, Xawery Żukow
2JSC “Dnipro-Beskyd”, Truskavets', Ukraine
${ }^{3}$ Ivan Franko Pedagogical University, Drohobych, Ukraine igorf3007@ukr.net doctor-0701@ukr.net oksanafigura08@gmail.com
${ }^{4} I Y$ Horbachevs'kyi National Medical University, Ternopil', Ukraine darakoz@yahoo.com \\ ${ }^{1}$ SE Ukrainian Research Institute for Medicine of Transport, Odesa, Ukraine \\ ${ }^{5}$ Medical University of Bialystok, Poland xaweryzukow@gmail.com
}

Background. Despite the long history, the results of studies of the impact of balneotherapy in the resort of Truskavets on the lithogenicity of urine are ambiguous, so the topic remains relevant. The aim of this study was to determine the relationship between the lithogenicity index of urine and a number of components of its composition. Materials and Methods. Experiment was performed on 60 healthy old female Wistar rats 220-300 g. Ten animals remained intact, using daily water from drinking ad libitum. Other animals for 6 days were loaded through the tube with daily and various mineral waters at a dose of $1,5 \mathrm{~mL} / 100 \mathrm{~g}$ of body mass. The day after the completion of the drinking course collected daily urine, which determined the content of a number of components of the composition. We calculated urine lithogenicity index (Lith) by the formula: Lith = (Uric acid $\cdot$ Calcium/Magnesium $\cdot$ Creatinine $)^{0,25}$. Results. The most significant effect on the Lith is the concentration of magnesium $(\mathrm{r}=-0,730)$, followed by uric acid $(\mathrm{r}=0,583)$, calcium $(\mathrm{r}=0,352)$ and creatinine $(\mathrm{r}=-0,298)$. Medium molecular polypeptides, catalase, sodium, phosphates and urea has been identified as prolithogenic factors while tubular reabsorption of water as litholytic factor. The chemical composition of the fluids consumed by animals has little effect on the lithogenicity index of urine. Conclusion. Both prolithogenic and litholytic factors are present in the urine, which depend little on the chemical composition of the fluid used.

Keywords: urine lithogenicity, drinking mineral waters, Truskavets' spa, female rats. 


\section{INRODUCTION}

Despite the long history, the results of studies of the impact of balneotherapy in the Truskavets' spa on the lithogenicity of urine are ambiguous [3-6,11,15-17], so the topic remains relevant. The aim of this study was to determine the relationship between the lithogenicity index of urine and a number of components of its composition.

\section{MATERIALS AND METHODS}

Experiment was performed on 60 healthy old female Wistar rats 220-300 g. Ten animals remained intact, using daily water from drinking ad libitum. Other animals for 6 days were loaded through the tube with daily and mineral waters (composition is given in Table 1) at a dose of $1,5 \mathrm{~mL} / 100 \mathrm{~g}$ of body mass.

Table 1. Chemical composition of fresh and mineral waters (according to the Truskavetsian Hydrogeological Regime-operational station)

\begin{tabular}{|c|c|c|c|c|c|}
\hline & Daily Water & Sofiya & Hertsa & Salt analog & Naftussya \\
\hline \multicolumn{6}{|c|}{ Electrolytes, $\mathrm{mM} / \mathrm{L}$} \\
\hline $\mathrm{Na}^{+}$ & 0,5 & 156 & 196,7 & 196,7 & 0,6 \\
\hline $\mathrm{Cl}^{-}$ & 3,4 & 142 & 205 & 205 & 1,0 \\
\hline $\mathrm{HCO}_{3}^{-}$ & 2,9 & 7,5 & 5,6 & 5,6 & 8,2 \\
\hline $\mathrm{Ca}^{2+}$ & 3,4 & 5,3 & 3,40 & 3,40 & 2,9 \\
\hline $\mathrm{Mg}^{2+}$ & 0,5 & 4,3 & 3,44 & 3,44 & 2,3 \\
\hline $\mathrm{K}^{+}$ & 0,4 & 0,3 & 0,4 & 0,4 & 0,3 \\
\hline $\mathrm{SO}_{4}{ }^{2-}$ & 1,2 & 13,1 & 0,1 & 0,1 & 1,0 \\
\hline \multicolumn{6}{|c|}{ Trace elements, mg/L } \\
\hline $\mathrm{H}_{2} \mathrm{SiO}_{3}$ & 5 & 4,43 & 9,88 & 0 & 9,5 \\
\hline $\mathrm{H}_{3} \mathrm{BO}_{3}$ & 0,25 & 8,39 & 42,76 & 0 & 0,200 \\
\hline $\mathrm{Br}$ & 8,3 & 6,7 & 21,17 & 0 & 0,034 \\
\hline $\mathrm{J}$ & 0,025 & 1,29 & 6,62 & 0 & 0,004 \\
\hline $\mathrm{F}$ & 0,95 & 0,52 & 0,57 & 0 & 0,160 \\
\hline \multicolumn{6}{|c|}{ Organic substances, $\mathrm{mg} / \mathrm{L}$} \\
\hline C org & 5,0 & 5,5 & 34 & 0 & 12,8 \\
\hline $\mathrm{N}$ org & 0,02 & 0,8 & 0,14 & 0 & 0,33 \\
\hline
\end{tabular}

The day after the completion of the drinking course animals were placed in individual chambers with perforated bottom for collecting daily urine. The content of the following substances in urine was determined. Electrolytes: calcium (by reaction with arsenase III), magnesium (by reaction with colgamite), phosphates (phosphate-molybdate method), chloride (mercury-rhodanidine method), sodium and potassium; nitric metabolites: creatinine (by Jaffe's color reaction by Popper's method), urea (urease method by reaction with phenolhypochlorite), uric acid (uricase method) and medium molecular polypeptides (by spectrophotometric method) [8]; lipid peroxidation components: diene conjugates (spectrophotometry of the heptane phase of the lipids extract [7]), malondyaldehide (in the test with thiobarbituric acid [1]) and catalase (at the rate of decomposition of hydrogen peroxide [14]), as well as amylase (Karavay's amyloclastic method with starch substrate) [8]. 
By the size of the diuresis and the level of creatinine in plasma and urine, glomerular filtration and tubular reabsorption were calculated.

Urine lithogenicity index (Lith) we calculated by the Tiselius' HS [18] formula modifed by Flyunt VR et al [5]: Lith $=(\text { Uric acid } \bullet \text { Calcium/Magnesium } \bullet \text { Creatinine })^{0,25}$.

The analyzes were carried out according to the instructions. The analyzers "Pointe-180" ("Scientific", USA) and "Reflotron" (Boehringer Mannheim, BRD) were used with appropriate sets and a flaming spectrophotometer "CФ-47".

Digital material is statistically processed on a computer using the software package "Statistica 64".

\section{RESULTS AND DISCUSSION}

For clarity, the urinary lithogenicity index was expressed as a Z-score:

$\mathrm{Z}=($ Variable/Intact -1$) / \mathrm{Cv})$.

Preliminary analysis revealed a wide range of lithogenicity values, and this applies even to intact animals (Fig. 1). Interestingly, among rats loaded with tap water, there were individuals with extreme levels of lithogenicity. Lithogenicity of rats of other groups also showed a pronounced variance.

This is consistent with the data of the Truskavets School of Balneology on the pronounced dispersion of the parameters of water-salt metabolism $[9,10]$ and the ambivalence of its reactions to the use of Naftussya water [2]. It seems that the level of lithogenicity of urine is not affected by water load as such or the chemical composition of fluids. The visual impression is generally confirmed by comparing the average values (Fig. 2), with the exception of Sofiya mineral water, which significantly reduces lithogenicity.

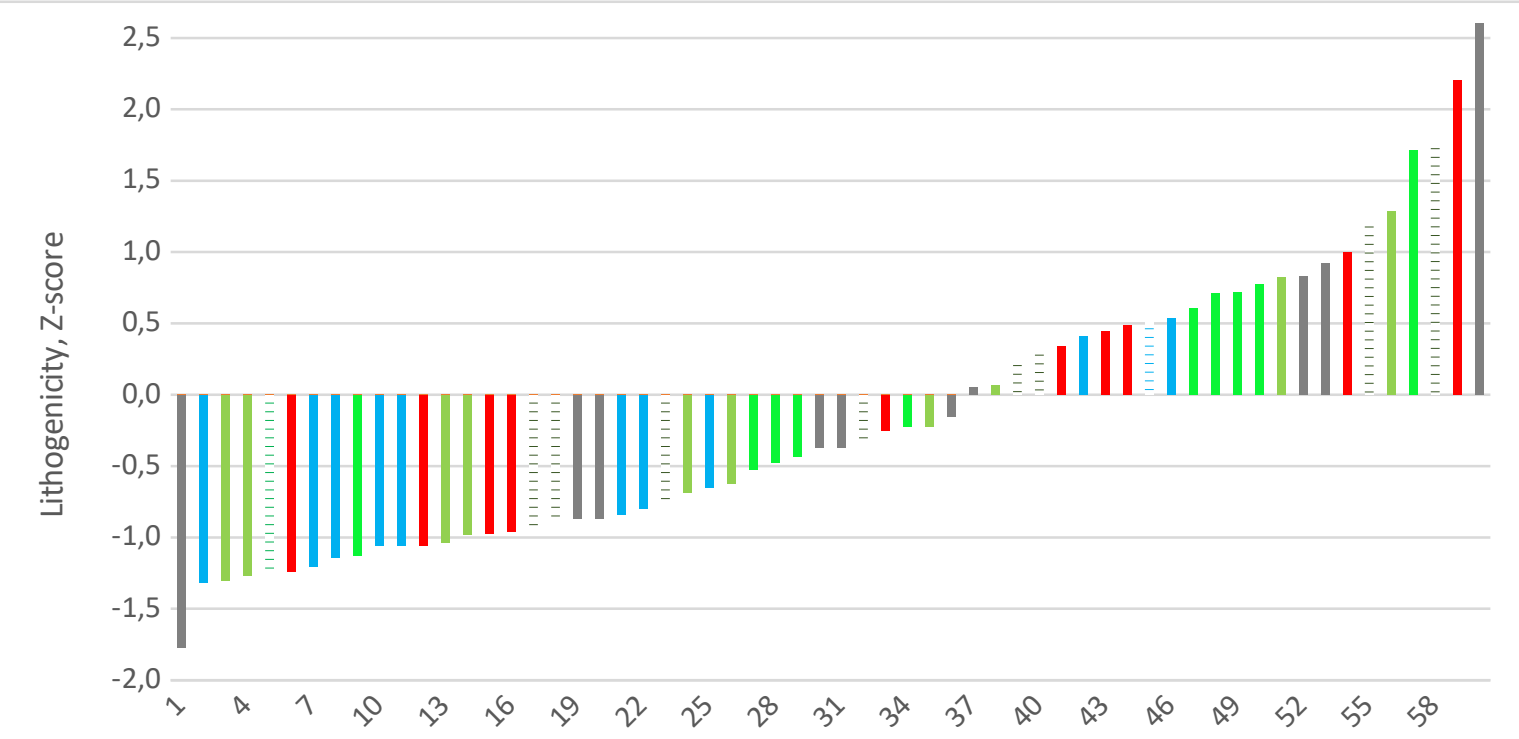

Fig. 1. Profile of urine lithogenicity index. Intact rats - columns with patterned filling, loaded with tap water - gray columns, Naftussya water - red, Sofiya water - blue, native Hertsa water - light green, its salt analog - green columns 


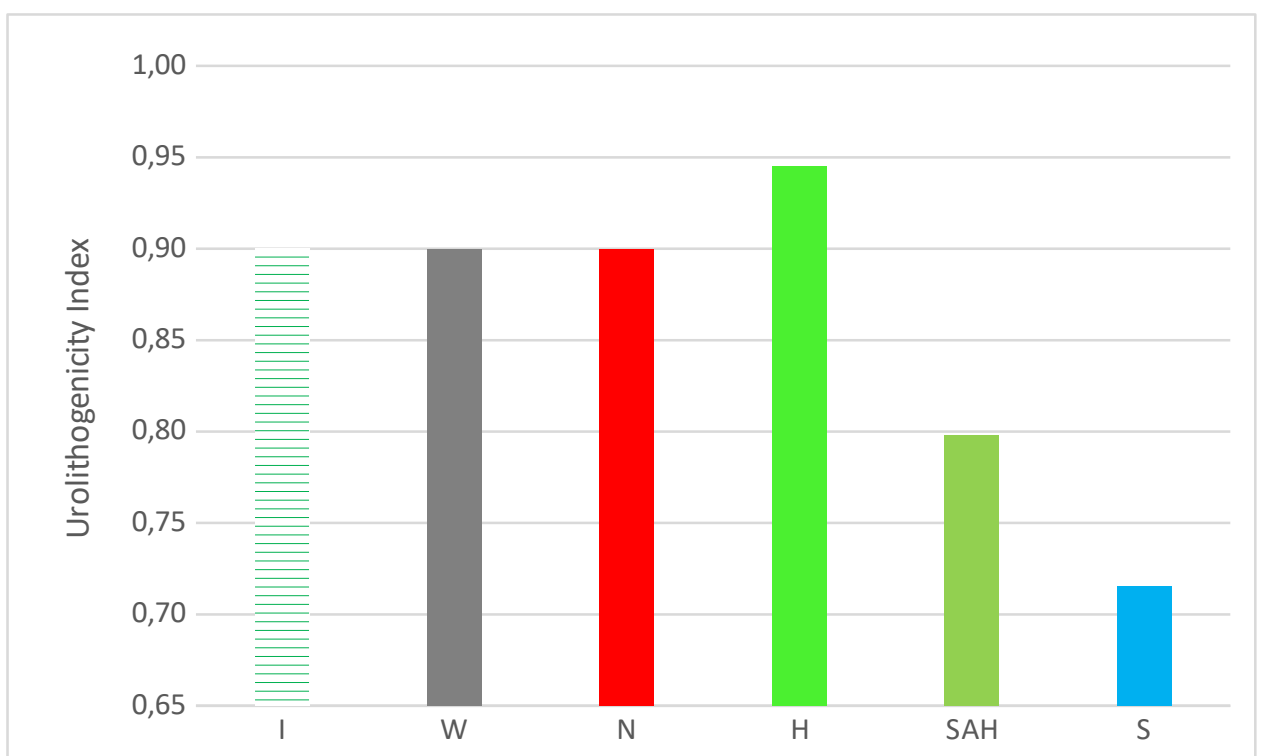

Fig. 2. Average values $($ Mean \pm SE) of urine lithogenicity index. Intact rats - column with patterned filling, loaded with tap water - gray, Naftussya water - red, native Hertsa water - light green, its salt analog - green, Sofiya water - blue columns

The difference in lithogenicity in rats loaded with native Hertza mineral water and its artificial salt analogue is noteworthy. Screening of correlations between lithogenicity and components of chemical composition of fluids consumed by animals revealed marginal significance (for a sample with $\mathrm{n}=60$ critical level $|\mathrm{r}|=0,255$ ) downregulating effects of calcium $(\mathrm{r}=-0,25)$ and sulfate $(\mathrm{r}=-0,25)$, as well as, to some extent, organic nitrogen $(\mathrm{r}=-0,21)$. Instead, the effects of organic carbon $(\mathrm{r}=0,21)$ and metasilicic acid $(\mathrm{r}=0.20)$ are upregulating. This at least partially clarifies the nature of the litholytic action of Sophia water and the prolithogenic action of Hertza native water.

At the next stage, the screening of correlations between the lithogenicity index of urine and its components - on the one hand, and other recorded parameters of urine and urination on the other hand. Only the coefficients worth noting are included in the matrix (Table 2). 
Table 2. Matrix of correlations between urine components

\begin{tabular}{|c|c|c|c|c|c|}
\hline \multirow[b]{2}{*}{ Variable } & \multicolumn{5}{|c|}{$\begin{array}{l}\text { Correlations } \\
\mathrm{N}=60\end{array}$} \\
\hline & Lith & $\mathrm{CrU}$ & $\mathrm{MgU}$ & $\mathrm{CaU}$ & UaU \\
\hline Lithogen & 1,00 & $-0,30$ & $-0,73$ & 0,35 & 0,58 \\
\hline $\mathrm{NaU}$ & 0,20 & $-0,19$ & $-0,17$ & 0,06 & 0,06 \\
\hline ClU & 0,02 & 0,00 & $-0,12$ & $-0,22$ & 0,06 \\
\hline Diuresis & 0,04 & $-0,25$ & $-0,22$ & $-0,07$ & $-0,26$ \\
\hline $\mathrm{CrU}$ & $-0,30$ & 1,00 & $-0,01$ & $-0,16$ & 0,08 \\
\hline GF & $-0,17$ & 0,18 & $-0,24$ & $-0,33$ & $-0,16$ \\
\hline CReabs & $-0,26$ & 0,64 & $-0,15$ & $-0,43$ & $-0,02$ \\
\hline UreaU & 0,17 & $-0,05$ & $-0,31$ & 0,23 & $-0,22$ \\
\hline KU & $-0,15$ & 0,28 & 0,03 & $-0,45$ & 0,20 \\
\hline $\mathrm{MgU}$ & $-0,73$ & $-0,01$ & 1,00 & 0,04 & $-0,30$ \\
\hline $\mathrm{CaU}$ & 0,35 & $-0,16$ & 0,04 & 1,00 & 0,00 \\
\hline PU & 0,19 & $-0,43$ & 0,06 & 0,32 & $-0,01$ \\
\hline $\mathrm{UaU}$ & 0,58 & 0,08 & $-0,30$ & 0,00 & 1,00 \\
\hline Amyl U & $-0,04$ & 0,39 & 0,07 & 0,00 & 0,21 \\
\hline MMMU & 0,36 & $-0,44$ & $-0,16$ & 0,24 & 0,05 \\
\hline KatalU & 0,34 & $-0,24$ & $-0,22$ & 0,39 & 0,07 \\
\hline MDAU & $-0,17$ & 0,36 & 0,05 & $-0,20$ & 0,07 \\
\hline $\mathrm{DCU}$ & $-0,08$ & 0,11 & $-0,07$ & $-0,19$ & $-0,12$ \\
\hline
\end{tabular}

The most significant effect on the lithogenicity index of urine is the concentration of magnesium in it (Fig. 3), followed by uric acid (Fig. 4), calcium (Fig. 5) and creatinine (Fig. $6)$.

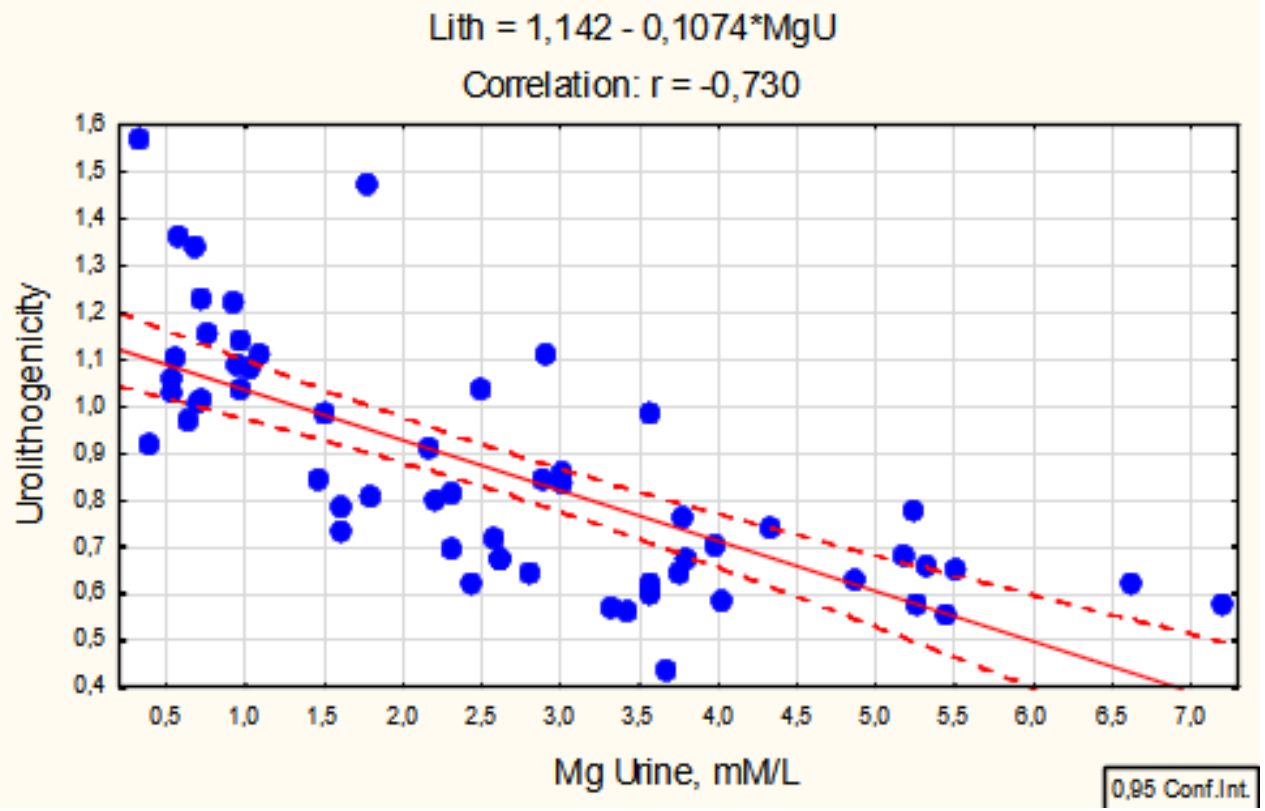

Fig. 3. Scatterplot of correlation between the magnesium urine (X-line) and lithogenicity index (Y-line) 


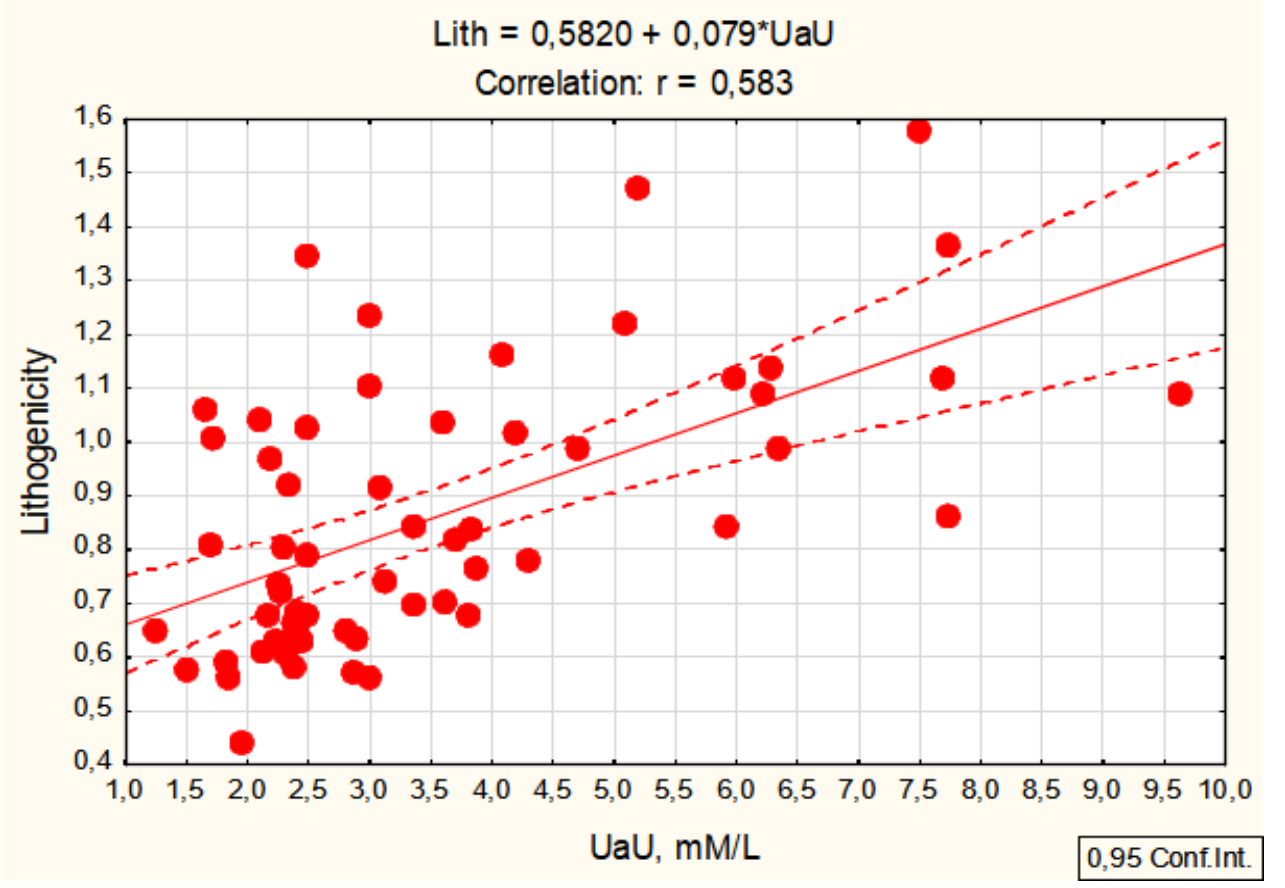

Fig. 4. Scatterplot of correlation between the uric acid urine (X-line) and lithogenicity index (Y-line)

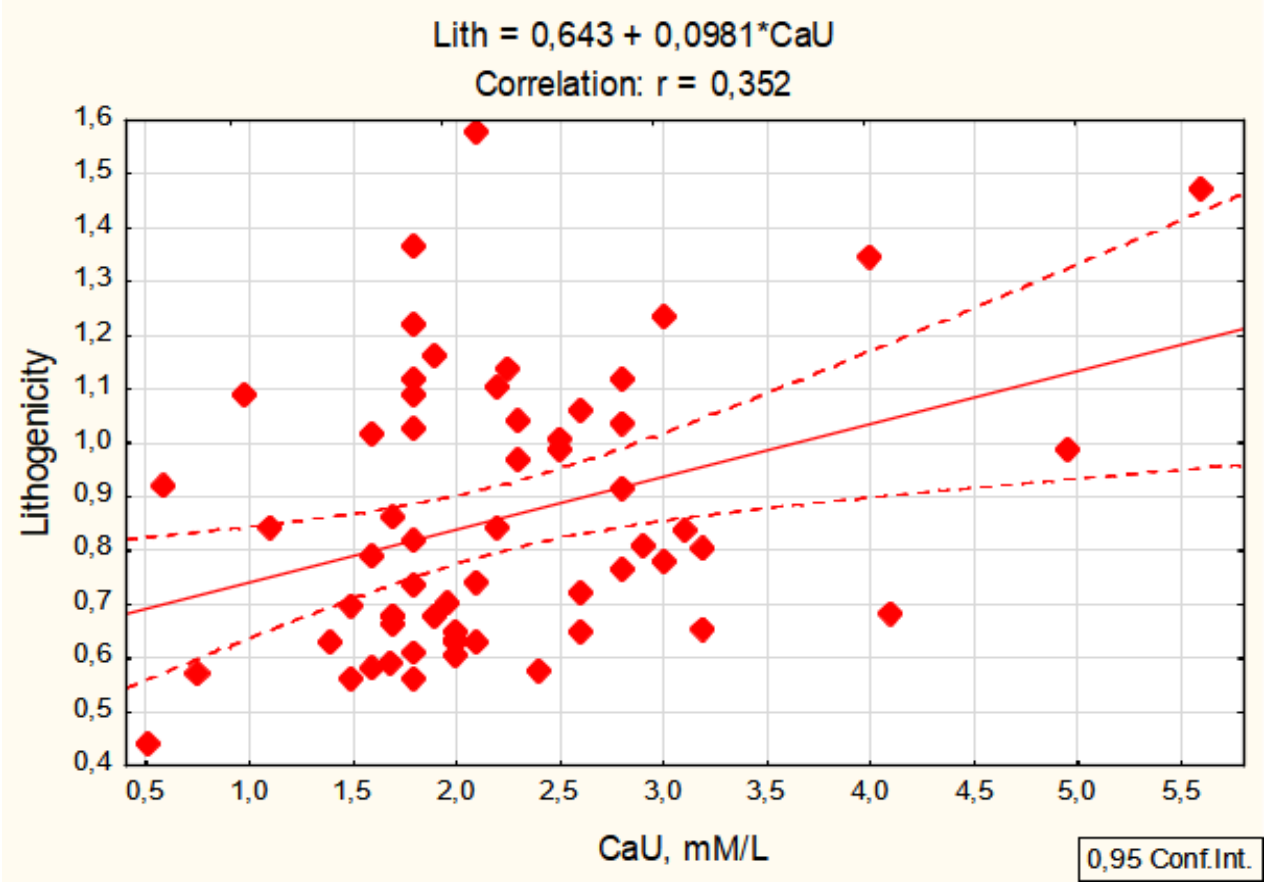

Fig. 5. Scatterplot of correlation between the calcium urine (X-line) and lithogenicity index (Y-line) 


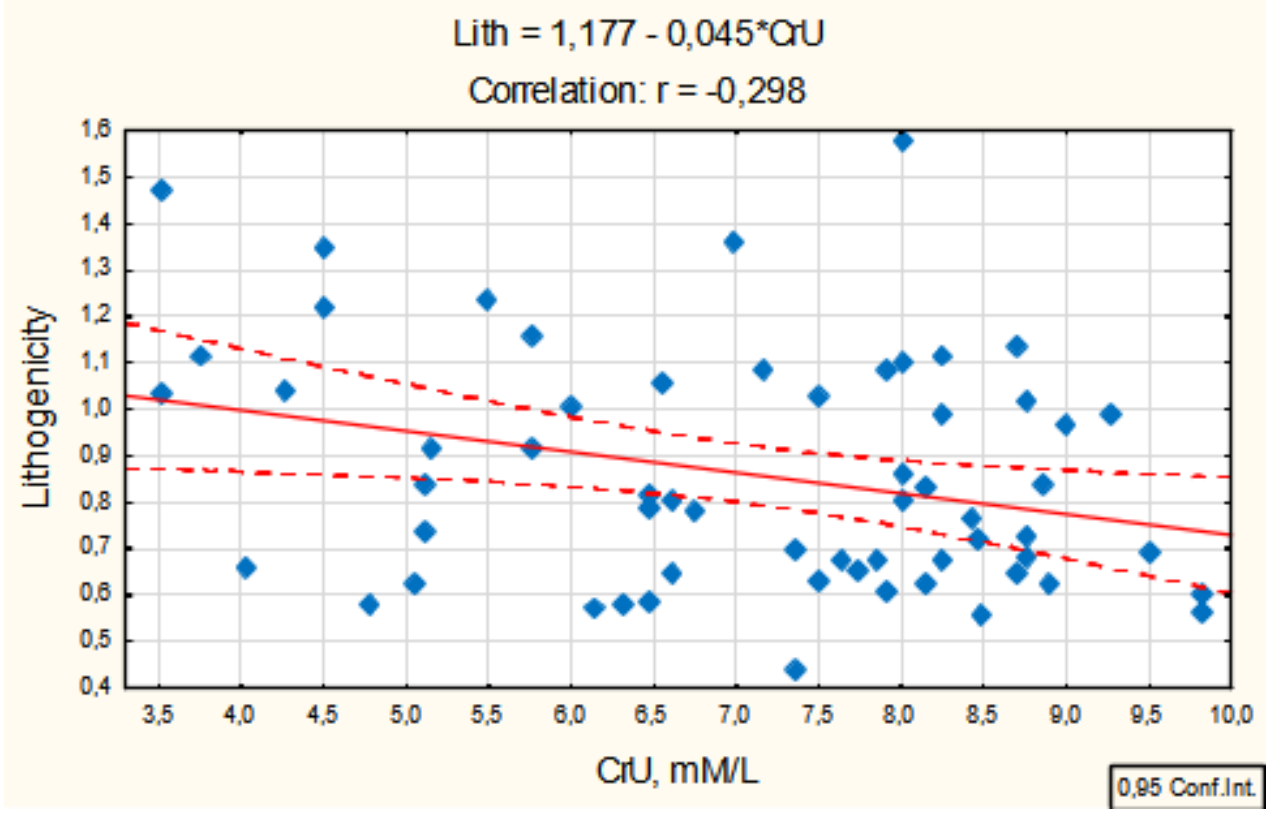

Fig. 6. Scatterplot of correlation between the creatinine urine (X-line) and lithogenicity index (Y-line)

Fig. 7 shows the relationship of the lithogenicity index with its major components, and in Fig. 8 - with all four, built on the parameters of the regression model (Table 3).

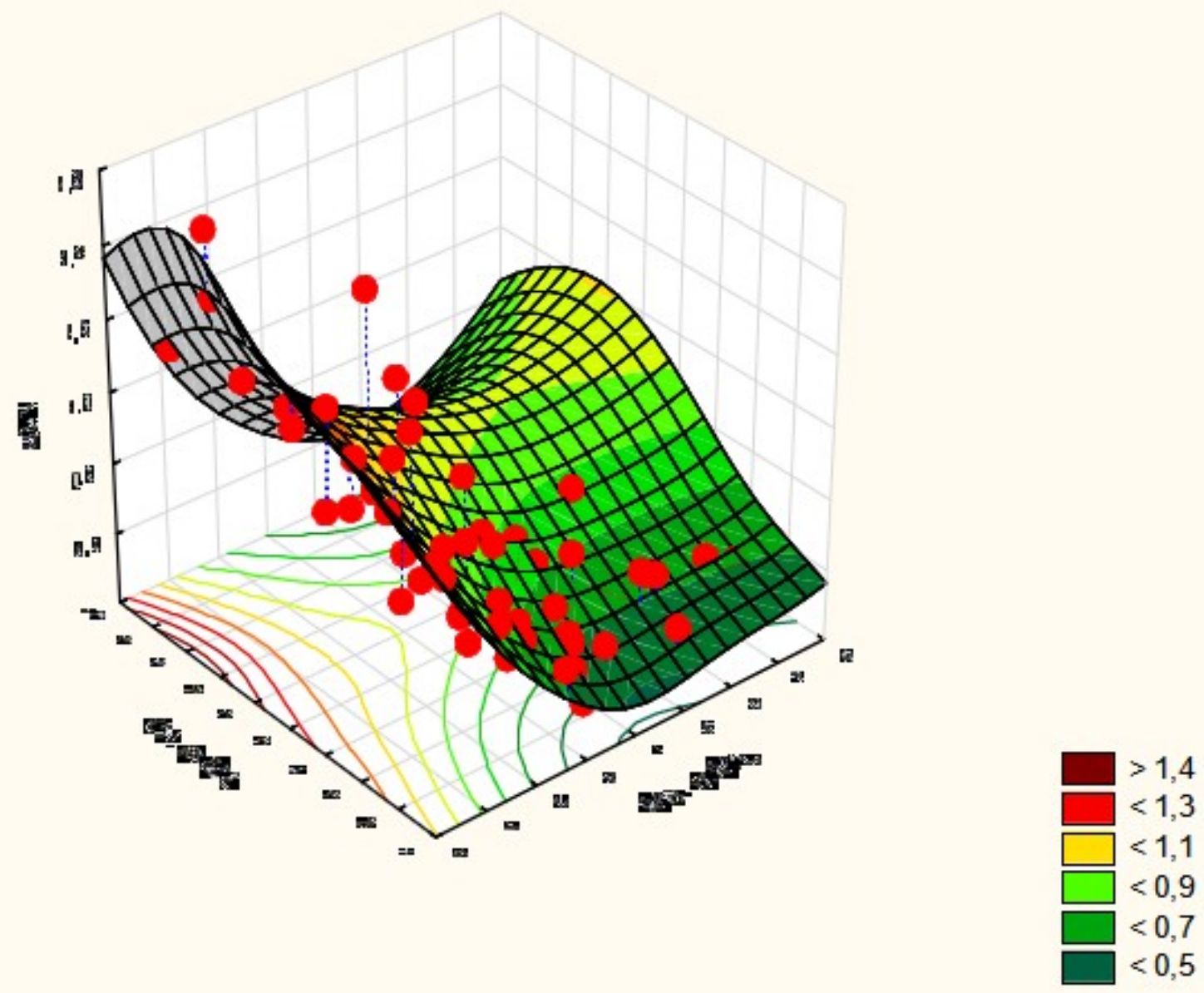

Fig. 7. Scatterplot of correlations between the urine magnesium (X-line), uric acid (Yline) (Y-line) and lithogenicity index (Z-line) 
Table 3. Regression Summary for Urolithogenicity index

$\mathrm{R}=0,947 ; \mathrm{R}^{2}=0,897 ;$ Adjusted $\mathrm{R}^{2}=0,890 ; \mathrm{F}_{(4,6)}=120 ; \mathrm{p}<10^{-4}$

\begin{tabular}{|l|l|l|l|l|l|l|l|}
\hline & & Beta & $\begin{array}{l}\text { St. Err. } \\
\text { of Beta }\end{array}$ & B & $\begin{array}{l}\text { SE } \\
\text { of B }\end{array}$ & $\mathrm{t}_{(55)}$ & $\begin{array}{l}\text { p- } \\
\text { level }\end{array}$ \\
\hline Variables & $\mathrm{r}$ & & Intercpt & 1,002 & 0,0647 & 15,5 & $10^{-6}$ \\
\hline Magnesium, mM/L & $\mathbf{- 0 , 7 3 0}$ & $-0,622$ & 0,045 & $-0,0914$ & 0,0067 & $-13,7$ & $10^{-6}$ \\
\hline Creatinine, mM/L & $\mathbf{- 0 , 2 9 8}$ & $-0,285$ & 0,044 & $-0,0428$ & 0,0066 & $-6,49$ & $10^{-6}$ \\
\hline Uric acid, mM/L & $\mathbf{0 , 5 8 3}$ & 0,415 & 0,045 & 0,0560 & 0,0061 & 9,13 & $10^{-6}$ \\
\hline Calcium, mM/L & $\mathbf{0 , 3 5 2}$ & 0,332 & 0,044 & 0,0923 & 0,0122 & 7,57 & $10^{-6}$ \\
\hline
\end{tabular}

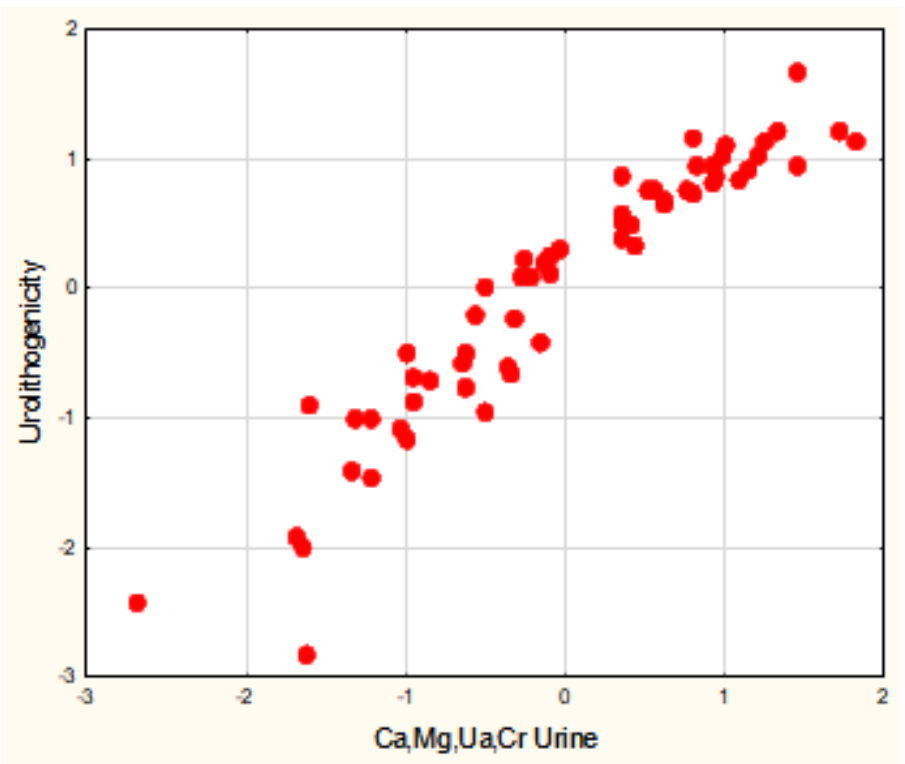

$\mathrm{R}=0,947 ; \mathrm{R}^{2}=0,897 ; \chi^{2}{ }_{(4)}=128 ; \mathrm{p}<10^{-6} ; \Lambda$ Prime $=0,103$

Fig. 8. Scatterplot of canonical correlation between urine lithogenic and litholytic substances (X-line) and urolithogenicity index (Y-line)

Next, the canonical correlation between the lithogenicity index of urine and all its registered parameters is analyzed. In the factor structure of urinary metabolic root, the program included, by definition, components of the lithogenicity index, medium molecular polypeptides, catalase, sodium, phosphates and urea as prolithogenic factors and tubular reabsorption of water as litholytic factor (Table 4).

Table 4. Factor structure of canonical roots of urine components

\begin{tabular}{|l|c|}
\hline \multirow{2}{*}{ Variable } & \\
\cline { 2 - 2 } Na U & Root 1 \\
\hline CReab & $-0,215$ \\
\hline Urea U & 0,278 \\
\hline P U & $-0,178$ \\
\hline MMM U & $-0,200$ \\
\hline Katal U & $-0,376$ \\
\hline Cr U & $-0,356$ \\
\hline Ca U & 0,314 \\
\hline Ua U & $-0,371$ \\
\hline Mg U & $-0,613$ \\
\hline
\end{tabular}

Interestingly, the additional inclusion of metabolic parameters in the factor structure of the urinary root had almost no effect on the value of the canonical correlation coefficient (Fig. 9). 
We interpret this as evidence of redundancy/duplication of information contained in these parameters.

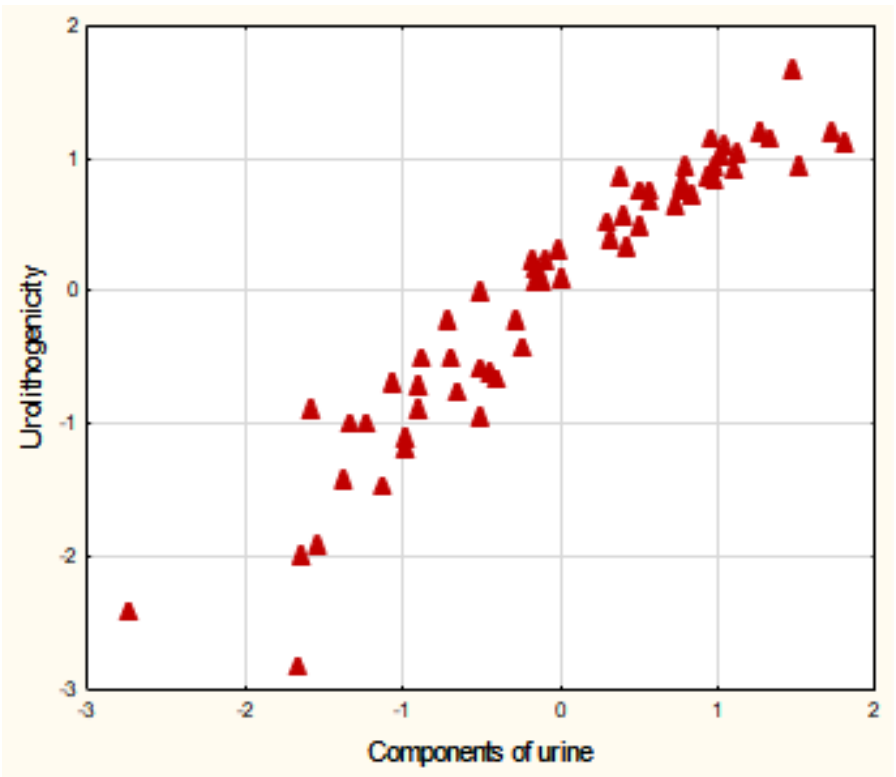

$\mathrm{R}=0,950 ; \mathrm{R}^{2}=0,902 ; \chi_{(10)}^{2}=123 ; \mathrm{p}<10^{-6} ; \Lambda$ Prime $=0,098$

Fig. 9. Scatterplot of canonical correlation between components of urine (X-line) and its lithogenicity (Y-line)

This statement is supported by the close links between the two sets of urinary metabolites (Table 5 and Fig. 10). 
Table 5. Results of the canonical correlation between two sets of urine components

\begin{tabular}{|l|c|}
\hline \multirow{2}{*}{ Variable } & \multicolumn{2}{|c|}{ Factor Structure, left set } \\
\cline { 2 - 2 } & Root 1 \\
\hline NaU & $-0,207$ \\
\hline CReab & 0,909 \\
\hline Urea U & $-0,094$ \\
\hline P U & $-0,623$ \\
\hline MMM U & $-0,559$ \\
\hline Katal U & $-0,425$ \\
\hline MDA U & 0,457 \\
\hline
\end{tabular}

\begin{tabular}{|c|c|c|c|c|}
\hline \multirow[b]{2}{*}{ Variable } & \multicolumn{3}{|c|}{ Factor Structure, right set } & \\
\hline & \multicolumn{2}{|c|}{ Root 1} & & \\
\hline $\mathrm{CrU}$ & \multicolumn{2}{|l|}{0,895} & & \\
\hline MgU & \multicolumn{2}{|l|}{$-0,117$} & & \\
\hline $\mathrm{CaU}$ & \multicolumn{2}{|l|}{$-0,563$} & & \\
\hline UaU & \multicolumn{2}{|l|}{$-0,006$} & & \\
\hline \multirow{2}{*}{$\begin{array}{l}\text { Root } \\
\text { Removed }\end{array}$} & \multicolumn{4}{|c|}{ Correlations, left set with right set } \\
\hline & $\mathrm{CrU}$ & $\mathrm{MgU}$ & $\mathrm{CaU}$ & UaU \\
\hline $\mathrm{NaU}$ & $-0,187$ & $-0,166$ & 0,059 & 0,064 \\
\hline CReab & 0,642 & $-0,146$ & $-0,426$ & $-0,016$ \\
\hline Urea U & $-0,047$ & $-0,308$ & 0,228 & $-0,219$ \\
\hline $\mathrm{PU}$ & $-0,435$ & 0,060 & 0,321 & $-0,014$ \\
\hline MMM U & $-0,438$ & $-0,162$ & 0,240 & 0,049 \\
\hline Katal U & $-0,236$ & $-0,219$ & 0,393 & 0,070 \\
\hline MDA U & 0,357 & 0,051 & $-0,200$ & 0,067 \\
\hline
\end{tabular}

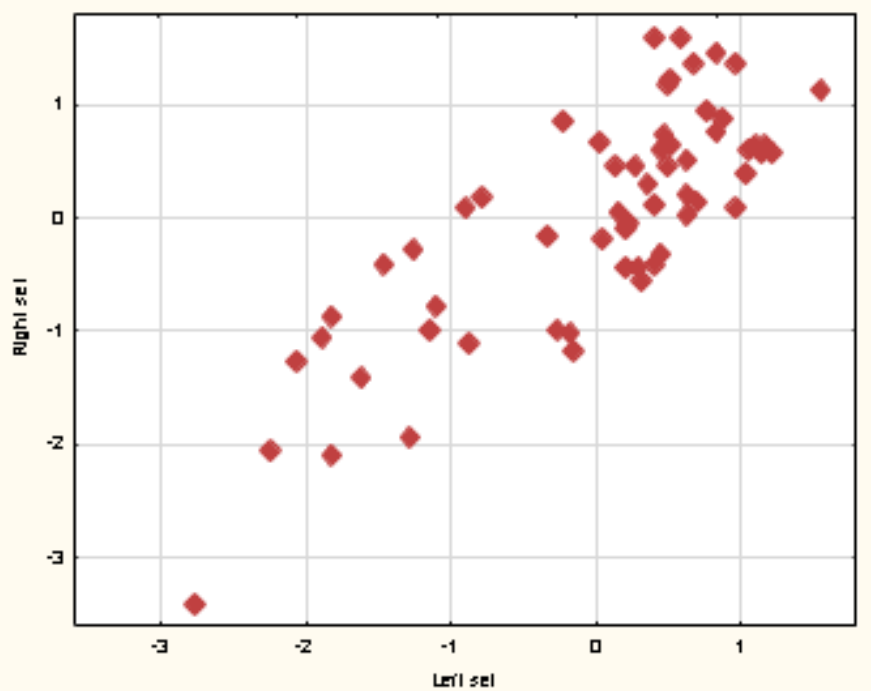

$\mathrm{R}=0,809 ; \mathrm{R}^{2}=0,655 ; \chi_{(28)}^{2}=85 ; \mathrm{p}<10^{-6} ; \Lambda$ Prime $=0,202$

Fig. 10. Scatterplot of canonical correlation between two sets of urine components

Another approach to achieve this goal is discriminant analysis (Tables 6-8). 
Table 6. Discriminant Function Analysis Summary

Step 8, N of Variables currently in the model: 8; Grouping: 3 groups

Wilks' Lambda: 0,1819; approx. $\mathrm{F}_{(16,1)}=8,40 ; \mathrm{p}<10^{-6}$

\begin{tabular}{|c|c|c|c|c|c|c|c|c|c|}
\hline \multirow[b]{2}{*}{$\begin{array}{l}\text { Variables } \\
\text { currently in the } \\
\text { model }\end{array}$} & \multicolumn{4}{|c|}{ Groups (n) } & \multicolumn{5}{|c|}{ Parameters of Wilks' Statistics } \\
\hline & $\begin{array}{l}\text { Intact } \\
\text { rats } \\
(10)\end{array}$ & $\begin{array}{l}\text { Lith - } \\
\text { (13) }\end{array}$ & $\begin{array}{l}\text { Lith } \pm \\
\text { (40) }\end{array}$ & $\begin{array}{l}\text { Lith + } \\
\text { (7) }\end{array}$ & $\begin{array}{l}\text { Wil } \\
\text { ks' } \\
\Lambda\end{array}$ & $\begin{array}{l}\text { Par- } \\
\text { tial } \\
\Lambda\end{array}$ & $\begin{array}{l}\text { F-re- } \\
\text { move } \\
(2,50)\end{array}$ & $\begin{array}{l}\text { p- } \\
\text { level }\end{array}$ & $\begin{array}{l}\text { Tole- } \\
\text { rancy }\end{array}$ \\
\hline $\begin{array}{l}\text { Magnesium } \\
\text { Urine, } \\
\mathrm{mM} / \mathrm{L}\end{array}$ & $\begin{array}{l}2,56 \\
1 \\
0\end{array}$ & $\begin{array}{l}4,38 \\
1,71 \\
+1,02\end{array}$ & $\begin{array}{l}2,38 \\
0,93 \\
-0,10\end{array}$ & $\begin{array}{l}0,82 \\
0,32 \\
-0,98\end{array}$ & 0,384 & 0,473 & 27,8 & $10^{-6}$ & 0,623 \\
\hline $\begin{array}{l}\text { Phosphates } \\
\text { Urine, } \\
\text { mM/L }\end{array}$ & $\begin{array}{l}6,39 \\
1 \\
0\end{array}$ & $\begin{array}{l}5,55 \\
0,87 \\
-1,07\end{array}$ & $\begin{array}{l}6,34 \\
0,99 \\
-0,06\end{array}$ & $\begin{array}{l}6,69 \\
1,05 \\
+0,38\end{array}$ & 0,221 & 0,822 & 5,42 & 0,007 & 0,693 \\
\hline $\begin{array}{l}\text { Katalase Activity } \\
\text { Urine, } \\
\mu \mathrm{M} / \mathrm{h} \cdot \mathrm{L}\end{array}$ & $\begin{array}{l}123 \\
1 \\
0\end{array}$ & $\begin{array}{l}129 \\
1,05 \\
+0,24\end{array}$ & $\begin{array}{l}134 \\
1,09 \\
+0,42\end{array}$ & $\begin{array}{l}204 \\
1,66 \\
+2,96\end{array}$ & 0,210 & 0,866 & 3,88 & 0,027 & 0,800 \\
\hline $\begin{array}{l}\text { Amylase Activity } \\
\text { Urine, } \\
\text { g/h॰L }\end{array}$ & $\begin{array}{l}202 \\
1 \\
0\end{array}$ & $\begin{array}{l}187 \\
0,92 \\
-0,28\end{array}$ & $\begin{array}{l}216 \\
1,07 \\
+0,25\end{array}$ & $\begin{array}{l}181 \\
0,89 \\
-0,40\end{array}$ & 0,208 & 0,875 & 3,58 & 0,035 & 0,703 \\
\hline $\begin{array}{l}\text { Calcium } \\
\text { Urine, } \\
\mathrm{mM} / \mathrm{L}\end{array}$ & $\begin{array}{l}2,10 \\
1 \\
0\end{array}$ & $\begin{array}{l}1,66 \\
0,79 \\
-1,17\end{array}$ & $\begin{array}{l}2,27 \\
1,08 \\
+0,47\end{array}$ & $\begin{array}{l}2,89 \\
1,38 \\
+2,10\end{array}$ & 0,228 & 0,799 & 6,27 & 0,004 & 0,574 \\
\hline $\begin{array}{l}\text { Uric Acid } \\
\text { Urine, } \\
\mathrm{mM} / \mathrm{L}\end{array}$ & $\begin{array}{l}3,68 \\
1 \\
0\end{array}$ & $\begin{array}{l}2,29 \\
0,62 \\
-0,75\end{array}$ & $\begin{array}{l}3,67 \\
1,00 \\
0,00\end{array}$ & $\begin{array}{l}5,02 \\
1,36 \\
+0,73\end{array}$ & 0,205 & 0,887 & 3,19 & 0,049 & 0,869 \\
\hline $\begin{array}{l}\text { Creatinine } \\
\text { Urine, } \\
\mathrm{mM} / \mathrm{L}\end{array}$ & $\begin{array}{l}6,41 \\
1 \\
0\end{array}$ & $\begin{array}{l}7,44 \\
1,16 \\
+0,56\end{array}$ & $\begin{array}{l}7,24 \\
1,13 \\
+0,45\end{array}$ & $\begin{array}{l}5,54 \\
0,86 \\
-0,47\end{array}$ & 0,205 & 0,889 & 3,13 & 0,052 & 0,655 \\
\hline $\begin{array}{l}\text { Potassium } \\
\text { Urine, } \\
\mathrm{mM} / \mathrm{L}\end{array}$ & $\begin{array}{l}131 \\
1 \\
0\end{array}$ & $\begin{array}{l}122 \\
0,94 \\
-0,21\end{array}$ & $\begin{array}{l}125 \\
0,96 \\
-0,14\end{array}$ & $\begin{array}{l}104 \\
0,80 \\
-0,68\end{array}$ & 0,190 & 0,958 & 1,10 & 0,340 & 0,671 \\
\hline
\end{tabular}

Note. In each column, the first line is the average value, the second is the fraction of the norm, and the third is the Z-score. 
Table 7. Discriminant Function Analysis Summary. Variables currently not in the model

\begin{tabular}{|c|c|c|c|c|c|c|c|c|c|}
\hline \multirow[b]{2}{*}{ Variables } & \multicolumn{4}{|c|}{ Groups (n) } & \multicolumn{5}{|c|}{ Parameters of Wilks' Statistics } \\
\hline & $\begin{array}{l}\text { Intact } \\
\text { rats } \\
(10)\end{array}$ & $\begin{array}{l}\text { Lith- } \\
\text { (13) }\end{array}$ & $\begin{array}{l}\text { Lith } \pm \\
\text { (40) }\end{array}$ & $\begin{array}{l}\text { Lith+ } \\
\text { (7) }\end{array}$ & $\begin{array}{l}\text { Wil } \\
\text { ks' } \\
\Lambda\end{array}$ & $\begin{array}{l}\text { Par- } \\
\text { tial } \\
\Lambda\end{array}$ & $\begin{array}{l}\text { F to } \\
\text { en- } \\
\text { ter }\end{array}$ & $\begin{array}{l}\mathrm{p}- \\
\text { level }\end{array}$ & $\begin{array}{l}\text { Tole- } \\
\text { rancy }\end{array}$ \\
\hline $\begin{array}{l}\text { Sodium } \\
\text { Urine, } \\
\mathrm{mM} / \mathrm{L}\end{array}$ & $\begin{array}{l}105 \\
1 \\
0\end{array}$ & $\begin{array}{l}62 \\
0,59 \\
-0,65\end{array}$ & $\begin{array}{l}108 \\
1,02 \\
+0,04\end{array}$ & $\begin{array}{l}125 \\
1,19 \\
+0,30\end{array}$ & 0,178 & 0,981 & 0,48 & 0,624 & 0,938 \\
\hline $\begin{array}{l}\text { Chloride } \\
\text { Urine, } \\
\mathrm{mM} / \mathrm{L}\end{array}$ & $\begin{array}{l}115 \\
1 \\
0\end{array}$ & $\begin{array}{l}88 \\
0,77 \\
-0,33\end{array}$ & $\begin{array}{l}118 \\
1,03 \\
+0,04\end{array}$ & $\begin{array}{l}100 \\
0,87 \\
-0,19\end{array}$ & 0,179 & 0,982 & 0,45 & 0,643 & 0,798 \\
\hline $\begin{array}{l}\text { Diuresis, } \\
\mathrm{mL} / 24 \mathrm{~h} \cdot 100 \mathrm{~g}\end{array}$ & $\begin{array}{l}1,44 \\
1 \\
0\end{array}$ & $\begin{array}{l}1,87 \\
1,30 \\
+0,48\end{array}$ & $\begin{array}{l}1,49 \\
1,04 \\
+0,06\end{array}$ & $\begin{array}{l}2,32 \\
1,61 \\
+0,99\end{array}$ & 0,177 & 0,974 & 0,66 & 0,523 & 0,573 \\
\hline $\begin{array}{l}\text { Glomerular } \\
\text { Filtration, } \\
\mu \mathrm{L} / \mathrm{min} \cdot 100 \mathrm{~g}\end{array}$ & $\begin{array}{l}86,0 \\
1 \\
0\end{array}$ & $\begin{array}{l}143 \\
1,66 \\
+1,85\end{array}$ & $\begin{array}{l}117 \\
1,37 \\
+1,02\end{array}$ & $\begin{array}{l}90,7 \\
1,05 \\
+0,15\end{array}$ & 0,177 & 0,975 & 0,62 & 0,541 & 0,663 \\
\hline $\begin{array}{l}\text { Canalicular } \\
\text { Reabsorbtion, } \\
\%\end{array}$ & $\begin{array}{l}98,69 \\
1 \\
0\end{array}$ & $\begin{array}{l}98,92 \\
1,002 \\
+0,28\end{array}$ & $\begin{array}{l}98,83 \\
1,001 \\
+0,17\end{array}$ & $\begin{array}{l}98,02 \\
0,993 \\
-0,83\end{array}$ & 0,178 & 0,976 & 0,59 & 0,558 & 0,455 \\
\hline $\begin{array}{l}\text { Urea } \\
\text { Urine, } \\
\mathrm{mM} / \mathrm{L}\end{array}$ & $\begin{array}{l}105 \\
1 \\
0\end{array}$ & $\begin{array}{l}100 \\
0,95 \\
-0,13 \\
\end{array}$ & $\begin{array}{l}124 \\
1,18 \\
+0,47 \\
\end{array}$ & $\begin{array}{l}142 \\
1,35 \\
+0,93 \\
\end{array}$ & 0,181 & 0,996 & 0,09 & 0,913 & 0,657 \\
\hline $\begin{array}{l}\text { Middle Mass } \\
\text { Molecules Urine, } \\
\text { units }\end{array}$ & $\begin{array}{l}182 \\
1 \\
0\end{array}$ & $\begin{array}{l}149 \\
0,82 \\
-0,62\end{array}$ & $\begin{array}{l}163 \\
0,89 \\
-0,37\end{array}$ & $\begin{array}{l}209 \\
1,15 \\
+0,53 \\
\end{array}$ & 0,179 & 0,985 & 0,38 & 0,685 & 0,832 \\
\hline $\begin{array}{l}\text { Malondialdehyde } \\
\text { Urine, } \\
\mu \mathrm{M} / \mathrm{L}\end{array}$ & $\begin{array}{l}92 \\
1 \\
0 \\
\end{array}$ & $\begin{array}{l}94 \\
1,02 \\
+0,04 \\
\end{array}$ & $\begin{array}{l}93 \\
1,01 \\
+0,03 \\
\end{array}$ & $\begin{array}{l}67 \\
0,73 \\
-0,58 \\
\end{array}$ & 0,179 & 0,981 & 0,46 & 0,632 & 0,747 \\
\hline $\begin{array}{l}\text { Diene conjugates } \\
\text { Urine, } \\
\mathrm{E}^{232} / \mathrm{mL}\end{array}$ & $\begin{array}{l}1,86 \\
1 \\
0 \\
\end{array}$ & $\begin{array}{l}1,87 \\
1,01 \\
+0,03 \\
\end{array}$ & $\begin{array}{l}1,78 \\
0,96 \\
-0,12 \\
\end{array}$ & $\begin{array}{l}1,58 \\
0,85 \\
-0,42\end{array}$ & 0,178 & 0,977 & 0,57 & 0,567 & 0,928 \\
\hline
\end{tabular}

\section{Table 8. Summary of Stepwise Analysis}

\begin{tabular}{|l|c|c|c|c|c|}
\hline $\begin{array}{l}\text { Variables } \\
\text { currently in the model }\end{array}$ & $\begin{array}{l}\text { F to } \\
\text { enter }\end{array}$ & $\begin{array}{l}\text { p- } \\
\text { level }\end{array}$ & $\Lambda$ & $\begin{array}{l}\text { F- } \\
\text { value }\end{array}$ & $\begin{array}{l}\text { p- } \\
\text { level }\end{array}$ \\
\hline Magnesium Urine, $\mathbf{m M} / \mathbf{L}$ & 16,9 & $10^{-5}$ & 0,628 & 16,9 & $10^{-5}$ \\
\hline Phosphate Urine, $\mathbf{m M} / \mathbf{L}$ & 11,6 & $10^{-4}$ & 0,444 & 14,0 & $10^{-6}$ \\
\hline Katalase Activity Urine, $\boldsymbol{\mu M} / \mathbf{h} \cdot \mathbf{L}$ & 7,79 & 0,001 & 0,346 & 12,8 & $10^{-6}$ \\
\hline Amylase Activity Urine, $\mathbf{g} / \mathbf{h} \cdot \mathbf{L}$ & 6,14 & 0,004 & 0,282 & 11,9 & $10^{-6}$ \\
\hline Calcium Urine, $\mathbf{m M} / \mathbf{L}$ & 4,49 & 0,016 & 0,241 & 11,0 & $10^{-6}$ \\
\hline Uric Acid Urine, $\mathbf{m M} / \mathbf{L}$ & 3,76 & 0,030 & 0,211 & 10,2 & $10^{-6}$ \\
\hline Creatinine Urine, $\mathbf{m M} / \mathbf{L}$ & 2,80 & 0,070 & 0,190 & 9,43 & $10^{-6}$ \\
\hline Potassium Urine, $\mathbf{m M} / \mathbf{L}$ & 1,10 & 0,340 & 0,182 & 8,40 & $10^{-6}$ \\
\hline
\end{tabular}

The dividing information contained in 8 variables is condensed in 2 canonical discriminant roots (Table 9). The major root contains $87 \%$ of discriminative opportunities $\left(\mathrm{r}^{*}=0,860 ;\right.$ Wilks' $\left.\Lambda=0,1819 ; \chi_{(16)}^{2}=91 ; \mathrm{p}<10^{-6}\right)$ and the minor root $13 \%\left(\mathrm{r}^{*}=0,547\right.$; Wilks' $\left.\Lambda=0,7008 ; \chi_{(7)}^{2}=19 ; \mathrm{p}=0,008\right)$.

Table 9 shows standardized (normalized) and non-standardized (raw) coefficients for discriminant variables. The calculation of the discriminant root values for each animal as the sum of the products of raw coefficients to the individual values of discriminant variables 
together with the constant enables the visualization of each rat in the information space of the roots (Fig. 11).

\section{Table 9. Standardized and Raw Coefficients for Canonical Variables}

\begin{tabular}{|c|c|c|c|c|}
\hline Coefficients & \multicolumn{2}{|c|}{ Standardized } & \multicolumn{2}{|c|}{ Raw } \\
\hline Variables & Root 1 & Root 2 & Root 1 & Root 2 \\
\hline Magnesium Urine, mM/L & 1,056 & $-0,259$ & 0,760 & $-0,186$ \\
\hline Phosphate Urine, mM/L & $-0,508$ & 0,469 & $-0,583$ & 0,538 \\
\hline Katalase Activity Urine, $\mu \mathrm{M} / \mathrm{h} \cdot \mathrm{L}$ & $-0,155$ & $-0,708$ & $-0,004$ & $-0,019$ \\
\hline Amylase Activity Urine, $\mathrm{g} / \mathrm{h} \cdot \mathrm{L}$ & $-0,398$ & 0,451 & $-0,011$ & 0,013 \\
\hline Calcium Urine, $\mathrm{mM} / \mathrm{L}$ & $-0,675$ & 0,197 & $-0,792$ & 0,231 \\
\hline Uric Acid Urine, mM/L & $-0,386$ & $-0,259$ & $-0,222$ & $-0,149$ \\
\hline Creatinine Urine, mM/L & 0,431 & 0,329 & 0,268 & 0,204 \\
\hline Potassium Urine, mM/L & $-0,274$ & 0,157 & $-0,0062$ & 0,0035 \\
\hline & \multicolumn{2}{|c|}{ Constants } & 5,950 & $-4,643$ \\
\hline & \multicolumn{2}{|c|}{ Eigenvalues } & 2,852 & 0,427 \\
\hline \multicolumn{3}{|c|}{ Cumulative Proportions } & 0,870 & 1 \\
\hline
\end{tabular}

In the Table 10 together with discriminant variables are also variables that carry identifying/ separating information, but were outside the model due to its duplication/redundancy. For ease of comparison, the values of the variables are transformed into Z-scores.

Table 10. Factor Structure Matrix (Correlations Variables-Canonical Roots) and Means of Roots and Variables

\begin{tabular}{|l|c|c|l|l|l|}
\hline & \multicolumn{2}{|c|}{$\begin{array}{c}\text { Correlations } \\
\text { Variables-Roots }\end{array}$} & $\begin{array}{l}\text { Lith }+ \\
(\mathbf{7})\end{array}$ & $\begin{array}{l}\text { Lith } \pm \\
\mathbf{4 0}\end{array}$ & $\begin{array}{l}\text { Lith }- \\
(\mathbf{1 3})\end{array}$ \\
\hline \multicolumn{1}{|c|}{ Root 1 (87\%) } & $\mathbf{R 1}$ & $\mathbf{R 2}$ & $\mathbf{- 2 , 8 1}$ & $-0,41$ & $+\mathbf{2 , 7 8}$ \\
\hline Calcium Urine & $\mathbf{- 0 , 2 4 7}$ & $-0,071$ & $+\mathbf{2 , 1 0}$ & $+0,47$ & $\mathbf{- 1 , 1 7}$ \\
\hline Uric Acid Urine & $\mathbf{- 0 , 2 7 1}$ & $-0,070$ & $+\mathbf{0 , 7 3}$ & 0,00 & $\mathbf{- 0 , 7 5}$ \\
\hline Phosphates Urine & $\mathbf{- 0 , 2 5 0}$ & 0,112 & $+\mathbf{0 , 3 8}$ & $-0,06$ & $\mathbf{- 1 , 0 7}$ \\
\hline Katalase Activity Urine & $\mathbf{- 0 , 2 6 0}$ & $-0,709$ & $+\mathbf{2 , 9 6}$ & $+0,42$ & $+\mathbf{0 , 2 4}$ \\
\hline Glomerular Filtration & & & $+\mathbf{1 , 8 5}$ & $+1,02$ & $+\mathbf{0 , 1 5}$ \\
\hline Canalicular Reabsorbtion & & & $+\mathbf{0 , 2 8}$ & $+0,17$ & $\mathbf{- 0 , 8 3}$ \\
\hline Malondialdehyde Urine & & & $+\mathbf{0 , 0 4}$ & $+0,03$ & $\mathbf{- 0 , 5 8}$ \\
\hline Diene conjugates Urine & & & $+\mathbf{0 , 0 3}$ & $-0,12$ & $\mathbf{- 0 , 4 2}$ \\
\hline Magnesium Urine & $\mathbf{0 , 4 5 6}$ & $\mathbf{0 , 0 1 9}$ & $\mathbf{- 0 , 9 8}$ & $-0,10$ & $+1, \mathbf{0 2}$ \\
\hline Creatinine Urine & $\mathbf{0 , 1 5 5}$ & 0,380 & $\mathbf{- 0 , 4 7}$ & $+0,45$ & $+\mathbf{0 , 5 6}$ \\
\hline Sodium Urine & & & $\mathbf{- 0 , 6 5}$ & $+0,04$ & $+\mathbf{0 , 3 0}$ \\
\hline Urea Urine & & & $\mathbf{- 0 , 1 3}$ & $+0,47$ & $+\mathbf{0 , 9 3}$ \\
\hline Middle Mass Molecules & & & $\mathbf{- 0 , 6 2}$ & $-0,37$ & $+\mathbf{0 , 5 3}$ \\
\hline \multicolumn{1}{|c|}{ Root 2 (13\%) } & $\mathbf{R 1}$ & $\mathbf{R 2}$ & $\mathbf{- 1 , 3 7}$ & $+\mathbf{0 , 4 2}$ & $-0,56$ \\
\hline Amylase Activity Urine & $-0,063$ & $\mathbf{0 , 6 4 3}$ & $-0,40$ & $+\mathbf{0 , 2 5}$ & $-0,28$ \\
\hline Potassium Urine & 0,043 & $\mathbf{0 , 2 0 1}$ & $\mathbf{- 0 , 6 8}$ & $\mathbf{- 0 , 1 4}$ & $-0,21$ \\
\hline Chloride Urine & & & $-0,33$ & $+\mathbf{0 , 0 4}$ & $-0,19$ \\
\hline Diuresis & & & $+0,48$ & $+\mathbf{0 , 0 6}$ & $+0,99$ \\
\hline
\end{tabular}

The localization of the cluster of rats with high lithogenicity index in the extreme left zone of the major root axis (Fig. 11) reflects their maximally elevated levels of the calcium and uric acid, on the one hand, and maximally reduced levels of the magnesium and creatinine, by definition, as well as phosphates and catalase as prolithogenic factors and sodium, urea and middle mass molecules as litholytic factors. At the opposite pole of the axis are rats with reduced lithogenicity, and the intermediate position, of course, is a normal cluster, ie with lithogenicity indices in the range: $-\sigma \div+\sigma$. It should be noted that our data contradict the 
position that hypercalciuria and hyperphosphaturia associated with the production of reactive oxygen species. However, this occurs in the presence of urolithiasis [12].

Additional differentiation of rats occurs along the axis of the minor root, demonstrating that both reduced and increased urinary lithogenicity is accompanied by lower than normal amylase activity and potassium and chloride concentrations in combination with higher daily diuresis.

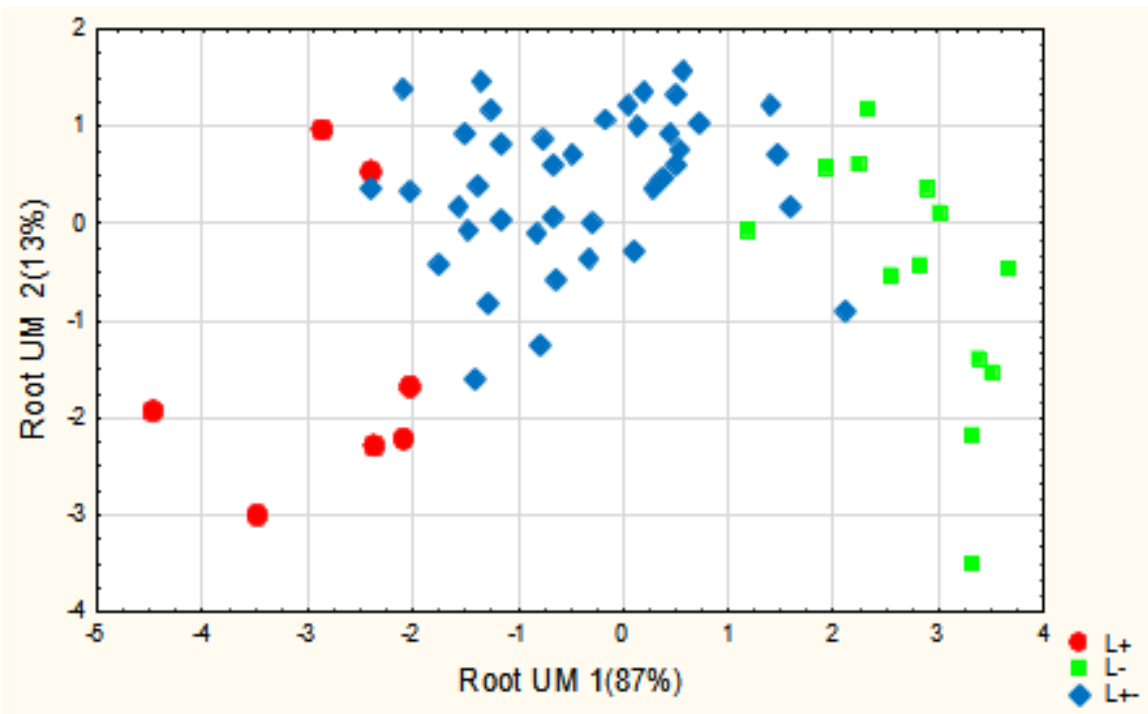

Fig. 11. Individual values of the first and second roots of the urine metabolic parameters in rats with different levels of lithogenicity

Despite separate interpenetrations, the three lithogenicity clusters differ significantly from each other, as documented by the calculation of Mahalanobis distances (Table 11).

Table 11. Squared Mahalanobis Distances between groups (over diagonal), F-values $(\mathrm{df}=\mathbf{8 , 5 0 )}$ and p-levels (under diagonal)

\begin{tabular}{|l|l|l|l|}
\hline Groups & $\begin{array}{l}\text { Lith }+ \\
(\mathbf{7})\end{array}$ & $\begin{array}{l}\text { Lith }- \\
(\mathbf{1 3})\end{array}$ & $\begin{array}{l}\text { Lith } \pm \\
\mathbf{( 4 0 )}\end{array}$ \\
\hline Lith + & $\mathbf{0 , 0}$ & 31,9 & 9,0 \\
\hline Lith - & $\begin{array}{l}\mathbf{1 5 , 9} \\
\mathbf{1 0}^{-6}\end{array}$ & $\mathbf{0 , 0}$ & 11,1 \\
\hline Lith \pm & $\begin{array}{l}\mathbf{5 , 9} \\
\mathbf{1 0}^{-4}\end{array}$ & $\begin{array}{l}\mathbf{1 2 , 0} \\
\mathbf{1 0}^{-6}\end{array}$ & $\mathbf{0 , 0}$ \\
\hline
\end{tabular}

The accuracy of the retrospective classification using the coefficients and constants of Table 12 is $93,3 \%$ (Table 13 ). 
Table 12. Coefficients and Constants for Classification Functions

\begin{tabular}{|l|c|c|c|}
\hline $\begin{array}{l}\text { Variables } \\
\text { currently in the model }\end{array}$ & $\begin{array}{c}\text { Lith }+ \\
(\mathbf{p}=\mathbf{0 , 1 1 7})\end{array}$ & $\begin{array}{c}\text { Lith }- \\
(\mathbf{p = 0 , 2 1 7 )}\end{array}$ & $\begin{array}{c}\text { Lith } \pm \\
(\mathbf{p}=\mathbf{0 , 6 6 7})\end{array}$ \\
\hline Magnesium Urine, mM/L & $-4,371$ & $-0,273$ & $-2,881$ \\
\hline Phosphate Urine, mM/L & 13,50 & 10,68 & 13,07 \\
\hline Katalase Activity Urine, $\boldsymbol{\mu M} / \mathbf{h} \cdot \mathbf{L}$ & 0,144 & 0,104 & 0,099 \\
\hline Amylase Activity Urine, $\mathbf{g} / \mathbf{h} \cdot \mathbf{L}$ & 0,192 & 0,138 & 0,187 \\
\hline Calcium Urine, mM/L & 5,091 & 0,854 & 3,606 \\
\hline Uric Acid Urine, mM/L & 1,854 & 0,489 & 1,053 \\
\hline Creatinine Urine, mM/L & 2,119 & 3,783 & 3,129 \\
\hline Potassium Urine, mM/L & 0,084 & 0,052 & 0,075 \\
\hline \multicolumn{1}{|c|}{ Constants } & $-99,75$ & $-68,78$ & $-87,33$ \\
\hline
\end{tabular}

Table 13. Classification Matrix

Rows: Observed classifications; Columns: Predicted classifications

\begin{tabular}{|c|c|c|c|c|}
\hline \multirow[t]{2}{*}{ Groups } & \multirow[t]{2}{*}{$\begin{array}{l}\text { Percent } \\
\text { correct }\end{array}$} & $\begin{array}{l}\text { Lith + } \\
\text { (7) }\end{array}$ & $\begin{array}{l}\text { Lith - } \\
\text { (13) }\end{array}$ & $\begin{array}{l}\text { Lith } \pm \\
(40)\end{array}$ \\
\hline & & $p=, 117$ & $\mathrm{p}=, 217$ & $\mathrm{p}=, 667$ \\
\hline Lith + (7) & 71,4 & 5 & 0 & 2 \\
\hline Lith - (13) & 92,3 & 0 & 12 & 1 \\
\hline Lith $\pm(40)$ & 97,5 & 0 & 1 & 39 \\
\hline Total & 93,3 & 5 & 13 & 42 \\
\hline
\end{tabular}

\section{CONCLUSION}

Both prolithogenic and litholytic factors are present in the urine, which depend little on the chemical composition of the fluid used.

\section{CONFORMITY TO ETHICAL STANDARDS}

Experiments on animals have been carried out in accordance with the provisions of the Helsinki Declaration of 1975, revised and supplemented in 2002 by the Directives of the National Committees for Ethics in Scientific Research.

The conduct of experiments was approved by the Ethics Committee of the Horbachevskyi Ternopil' National Medical University. The modern rules for the maintenance and use of laboratory animals complying with the principles of the European Convention for the Protection of Vertebrate Animals used for scientific experiments and needs are observed (Strasbourg, 1985).

\section{REFERENCES}

1. Andreyeva LI, Kozhemyakin LA, Kishkun AA. Modification of the method for determining the lipid peroxide in the test with thiobarbituric acid [in Russian]. Laboratornoye Delo. 1988; 11: 4143.

2. Balanovs'kyi VP, Popovych IL, Karpynets' SV. About ambivalence-equilibratory character of influence of curative water Naftussya on organism of human [in Ukrainian]. Dopovidi ANU. Mat pryr tekhn Nauky. 1993; 3: 154-158.

3. Flyunt IS, Fajda OI, Flyunt VR, Velychko LM, Tymochko OB, Hrebinyuk AI. Natural classification effects balneotherapy on spa Truskavets' in patients with oxalate urolithiasis. Communication 1: Level lithogenic and litholytic substances [in Ukrainian]. Medical Hydrology and Rehabilitation. 2004; 2(4): 53-56.

4. Flyunt VR, Flyunt IS, Barylyak LG, Piskov GG, Yanchiy OR. Links of urine $\mathrm{Ca} / \mathrm{Mg}$ coefficient of Lithogenicity of some parameters of electrolyte exchange in patient spa Truskavets' [in 
Ukrainian]. Medical Hydrology and Rehabilitation. 2013; 11(3): 43-49.

5. Flyunt VR, Flyunt IS, Popovych IL. Neuro-hormonal mechanism of action of bioactive water Naftussya on urine lithogenicity in rats [in Ukrainian]. Achievements of Clinical and Experimental Medicine. 2013; 2(19): 288.

6. Flyunt VR, Flyunt I-SS, Fil' VM, Kovbasnyuk MM, Hryvnak RF, Popel SL, Zukow W. Relationships between caused by drinking of bioactive water Naftussya changes in urine lithogenicity and neuro-humoral-immune factors in humans with their abnormalities. Journal of Education, Health and Sport. 2017; 7(3): 11-30.

7. Gavrilov VB, Mishkorudnaya MI. Spectrophotometric determination of plasma levels of lipid hydroperoxides [in Russian]. Laboratornoye Delo. 1983; 3: 33-36.

8. Goryachkovskiy AM. Clinical Biochemistry [in Russian]. Odesa. Astroprint; 1998: 608.

9. Hrytsan II, Gozhenko AI, Badiuk NS, Zukow W. Variants of the state of electrolyte exchange in female rates. In: Rehabilitation Medicine and Health-Resort Institutions Development. Proceedings of the 19th International Applied Research Conference (Kyïv, 11-12 December 2019). Edited by O. Gozhenko, W. Zukow. Toruń, Kyïv. 2019: 25-26.

10. Hrytsan II, Gozhenko AI, Badiuk NS, Zukow W. Variants of the state of electrolyte exchange in female rates. Journal of Education, Health and Sport. 2019; 9(10): 262-279.

11. Ivassivka SV, Popovych IL, Aksentiychuk BI, Flyunt IS. Physiological Activity of Uric Acid and its Role in the mechanism of action of Water Naftussya [in Ukrainian]. Kyiv. Computerpress; 2004: 163.

12. Khan SR, Canales BK, Dominguez-Gutierrez PR. Randall's plaque and calcium oxalate stone formation: role for immunity and inflammation. Nat Rev Nephrol. 2021; 17(6): 417-433.

13. Klecka WR. Discriminant Analysis [trans. from English in Russian] (Seventh Printing, 1986). In: Factor, Discriminant and Cluster Analysis. Moskva: Finansy i Statistika; 1989: 78-138.

14. Korolyuk MA, Ivanova MI, Mayorova IG, Tokarev VYe. The method for determining the activity of catalase [in Russian]. Laboratornoye Delo. 1988; 1: 16-19.

15. Shulyak OV, Flyunt IS, Flyunt VR, Fil' VM, Hrinchenko BV, Snihur OV, Zukow W, Yanchiy OR, Popovych IL. Influence of balneotherapy on spa Truskavets' with using Canephron ${ }^{\circledR} \mathrm{N}$ on urina lithogenicity and its metabolic and neuroendocrine-immune accompanyments in patients with chronic pyelonephrite and cholecystite. Journal of Education, Health and Sport. 2015; 5(11): 453-465.

16. Skorobogatov MO. Spa treatment of urolithiasis [in Ukrainian]. Kyiv. Zdorovya; 1991: 64.

17. Stetsenko HI, Beyda PA, Perchenko VP, Loyik IV, Flyunt IS, Yatsyuk VM. Restoration of the digestive and urinary excretion systems of victims of the Chernobyl disaster [in Ukrainian]. In: Resort Rehabilitation Victims of Chornobylian Accident. Kyiv. Zdorovya; 1999: 65-79.

18. Tiselius HS. A biochemical basis for grouping of patients with urolithiasis. Europ Urol. 1978; 4: 241-249. 